\title{
Robustness of networks against viruses: the role of the spectral radius
}

\author{
A. Jamakovic ${ }^{1}$, R.E. Kooij ${ }^{1,2}$, P. Van Mieghem ${ }^{1}$, E.R. van Dam ${ }^{3}$

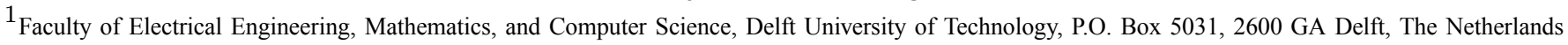

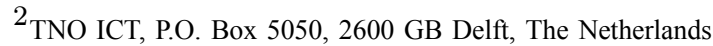

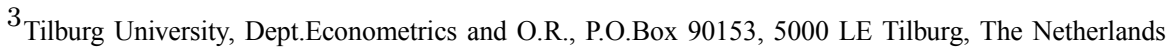

\begin{abstract}
In this paper we study the spectral radius of a number of real-life networks. This study is motivated by the fact that the smaller the spectral radius, the higher the robustness of a network against the spread of viruses. First we study how wellknown upper bounds for the spectral radius of graphs match to the spectral radii of the social network of the Dutch soccer team, the Dutch roadmap network, the network of the observable part of the Internet graph at the IP-level and the Autonomous System level. Secondly, we compare the spectral radius for these real-life networks with those of commonly used complex network models.
\end{abstract}

\section{INTRODUCTION}

The theory of the spectra of graphs contains many beautiful results, that relate physical properties of a network, such as for instance robustness, diameter and connectivity, to eigenvalues of matrices associated with the graph, see e.g. [6], [15]. Recently, it has been shown [16] that the spectral radius of a graph (i.e. the largest eigenvalue of its corresponding adjacency matrix) plays an important role in modeling virus propagation in networks. In fact, in [16], the SusceptibleInfected-Susceptible (SIS) infection model is considered. The SIS model assumes that a node in the network is in one of two states: infected and therefore infectious, or healthy and therefore susceptible to infection. The SIS model assumes instantaneous state transitions. Thus, as soon as a node becomes infected, it becomes infectious and likewise, as soon as a node is cured, it is susceptible to re-infection. Epidemiological theory [7] predicts the existence of an epidemic threshold $\tau$. If the infection rate along each link is $\beta$, while the cure rate for each node is $\delta$, then the effective spreading rate of the virus can be defined as $\beta / \delta$. The epidemic threshold can be defined as follows: for effective spreading rates below $\tau$ the virus contamination in the network dies out, while for effective spreading rates above $\tau$, the virus is prevalent, i.e. a persisting fraction of nodes remains infected. It was shown in [16] that $\tau=1 / \rho(G)$ where $\rho(G)$ denotes the spectral radius of the adjacency matrix of the graph $G$. If follows from this result that the smaller the spectral radius, the higher the robustness of a network against the spread of viruses.

The contribution of this paper is twofold. First, we study how well-known upper bounds for the spectral radius of graphs match the spectral radii of a number of real-world networks. Secondly, we compare the spectral radius of these real-world networks with those of commonly used network models.

\section{UPPER BOUNDS FOR THE SPECTRAL RADIUS}

An upper bound for the spectral radius of a graph [16] gives a lower bound for the epidemic threshold for virus propagation of the network. If the effective spreading rate is below this lower bound, then the virus contamination dies out. The sharper the upper bound for the spectral radius, the less effort we need to spend in reducing the effective spreading rate below the lower bound. The effective spreading rate can be lowered by either decreasing the spreading rate $\beta$ (e.g. by implementing more or better intrusion detection/prevention software) or by increasing the cure rate $\delta$ (e.g. by installing more virus scanning software).

The most common graphs for which an explicit expression for the spectral radius is known, are [6]: the complete graph $K_{n}$, the path $P_{n}$, the cycle graph $C_{n}$, the $k$-regular graph, the $k$-dimensional lattice and the complete bipartite graph $K_{m, n}$. Since no closed expression is known for the spectral radius of a general graph, we will discuss a number of upper bounds for the spectral radius of graphs. Although many more bounds are known, the most important (and best) ones are presented. The bounds differ both in form as in the parameters that are used. The more information about a graph is used, the better the bounds can be. Since different parameters of graphs are used in the bounds, it seems hard to compare them in general.

Now, let $G$ be a graph on $N$ nodes, with $L$ links, with minimum degree $d_{\min }$, maximum degree $d_{\max }$, and spectral radius $\rho(G)$. The oldest and simplest bound, that can be found in any book on spectral graph theory, is

$$
\rho(G) \leq d_{\max }
$$

A bound in terms of the numbers of nodes and links only is found by Hong [9]: if $G$ is connected, then

$$
\rho(G) \leq \sqrt{2 L-N+1}
$$

Cao [4] improved this bound at the cost of using more parameters: if $d_{\min } \geq 1$, then

$$
\rho(G) \leq \sqrt{2 L-(N-1) d_{\min }+\left(d_{\min }-1\right) d_{\max }}
$$

Hong, Shu, and Fang [10] obtained a bound that indicates the relation of the spectral radius to the minimal degree: if $G$ is connected, then 


$$
\rho(G) \leq \frac{1}{2}\left[d_{\min }-1+\sqrt{\left(d_{\min }+1\right)^{2}+4\left(2 L-N d_{\min }\right)}\right]
$$

Das and Kumar [8] obtained a bound that uses very local information of the graph: if $G$ is connected, and $m_{i}$ is the average degree of the nodes adjacent to node $i$, then

$$
\rho(G) \leq \max \left\{\sqrt{m_{i} m_{j}}: i \sim j\right\}
$$

Here $i \sim j$ indicates that nodes $i$ and $j$ are linked. Finally, Cioabă, Gregory, and Nikiforov [5] obtained an upper bound that also uses the diameter $D$ of the graph: if $G$ is connected and nonregular, then

$$
\rho(G)<d_{\max }-\frac{N d_{\max }-2 L}{N\left(D\left(N d_{\max }-2 L\right)+1\right)}
$$

\section{SPECTRAL RADIUS OF REAL-WORLD NETWORKS}

In this section, we give the spectral radius $\rho$ for the following real-world networks: the social network that is formed by all soccer players that have played an international match for the Dutch soccer team ${ }^{1}(\mathbf{A})$, the Dutch roadmap network (B) [11], the network of the observable part of the Internet graph at the IP-level (C) [12] and the Autonomous System level (D) [13].

An impression of the network of Dutch soccer team players is given in Figure 1. In this social network every node corresponds to a soccer player that has played a game for the Dutch national team. A node is connected with another node if both players have appeared in the same match.

For the considered real-world networks, we show in Table I, a set of generic topological characteristics. Furthermore, Table II illustrates the tightness of the upper bounds introduced in Section II.

We conclude from Table II that for networks A, B and C the upper bound (5) of Das and Kumar exhibits the best match with the real spectral radius. The overestimation of this upper bound for networks $\mathbf{A}, \mathbf{B}$ and $\mathbf{C}$ is $31 \%, 20 \%$ and $20 \%$, respectively. For network D, the upper bounds (2), (3) and (4) provide the best match. However, in this case, the overestimation is as high as $187 \%$. In addition, the following observations can be made from Table II:

- the upper bounds (2), (3) and (4) are of the same order

- the upper bound (6) does not give an improvement of upper bound (1)

- for networks $\mathbf{A}$ and $\mathbf{B}$, the simple upper bound (1) outperforms the upper bounds (2), (3) and (4).

Next, we explore the spectral radius of generic models (see e.g. [1] and [14]), used for modelling the evolution and the topology of real-world networks, i.e. the random graph of

\footnotetext{
${ }^{1}$ The data for the Dutch soccer team network was obtained through www.voetbalstats.nl, which gives the line-ups for all Dutch international soccer matches. We have considered all matches up till Ireland-Holland (16 august 2006), which was match number 644.
}

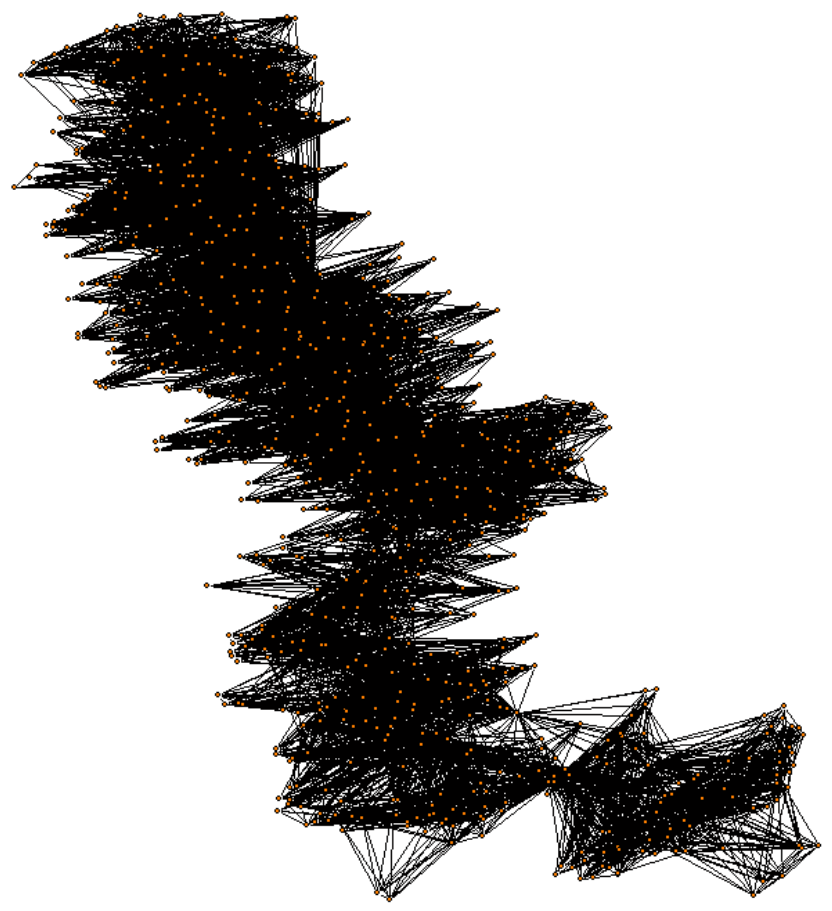

Fig. 1. The real-world network of Dutch soccer team players.

Erdös-Rényi (ER) [2], the small-world graph of Watts-Strogatz (WS) [17] and the scale-free graph of Barabási-Albert (BA) [3].

The ER graph is the most investigated topology model [2]. The most frequently occurring realization of this model is $G_{p}(N)$, in which $N$ is the number of nodes and $p$ is the probability that there is a link between any two nodes. The major characteristic of $G_{p}(N)$ is that the existence of a link is independent from the existence of other links. The total number of links in $G_{p}(N)$ is on average equal to $p L_{\max }$, where $L_{\max }=\left(\begin{array}{c}N \\ 2\end{array}\right)$ is the maximum possible number of links. Hence, the link density $q=\frac{L}{L_{\max }}$ equals $p$.

\begin{tabular}{|l|l|l|l|l|}
\hline $\begin{array}{l}\text { topological } \\
\text { characteristics }\end{array}$ & $\mathbf{A}$ & $\mathbf{B}$ & $\mathbf{C}$ & $\mathbf{D}$ \\
\hline$N$ & 685 & 14098 & 4058 & 18121 \\
\hline$L$ & 10271 & 18689 & 6151 & 59507 \\
\hline$d_{\max }$ & 117 & 6 & 107 & 2404 \\
\hline$d_{\min }$ & 9 & 1 & 2 & 1 \\
\hline $\bar{d}$ & 30 & 2.7 & 3 & 6.2 \\
\hline$D$ & 11 & 255 & 34 & 8 \\
\hline$\rho$ & 50.7 & 3.5 & 14.2 & 110.8 \\
\hline
\end{tabular}

\section{TABLE I}

TOPOLOGICAL CHARACTERISTICS OF THE REAL-WORLD NETWORK OF Dutch SOCCER TEAM Players (A), DUtCh ROADMAP (B), IP-LEVEL

(C) AND AS-LEVEl (D) InTERNET GRAPH. THE NOTATION WAS INTRODUCED IN THE PREVIOUS SECTION, WITH THE EXCEPTION OF $\bar{d}$ WHICH DENOTES AVERAGE NODAL DEGREE.

The WS graph captures the fact that, despite the large size of 


\begin{tabular}{|l|l|l|l|l|}
\hline upper bound & A & B & C & D \\
\hline$\rho$ & 50.7 & 3.5 & 14.2 & 110.8 \\
\hline 1 & 117 & 6 & 107 & 2404 \\
\hline 2 & 140.9 & 152.6 & 90.8 & 317.6 \\
\hline 3 & 123.8 & 152.6 & 65.5 & 317.6 \\
\hline 4 & 120.0 & 152.6 & 65.2 & 317.6 \\
\hline 5 & 66.6 & 4.2 & 17.1 & 1252.5 \\
\hline 6 & 117 & 6 & 107 & 2404 \\
\hline
\end{tabular}

TABLE II

UPPER BOUNDS ON THE SPECTRAL RADIUS OF THE REAL-WORLD NETWORK OF DUTCH SOCCER TEAM PLAYERS (A), DUTCH ROADMAP NETWORK (B), IP-LEVEL (C) AND AS-LEVEL (D) INTERNET GRAPH.

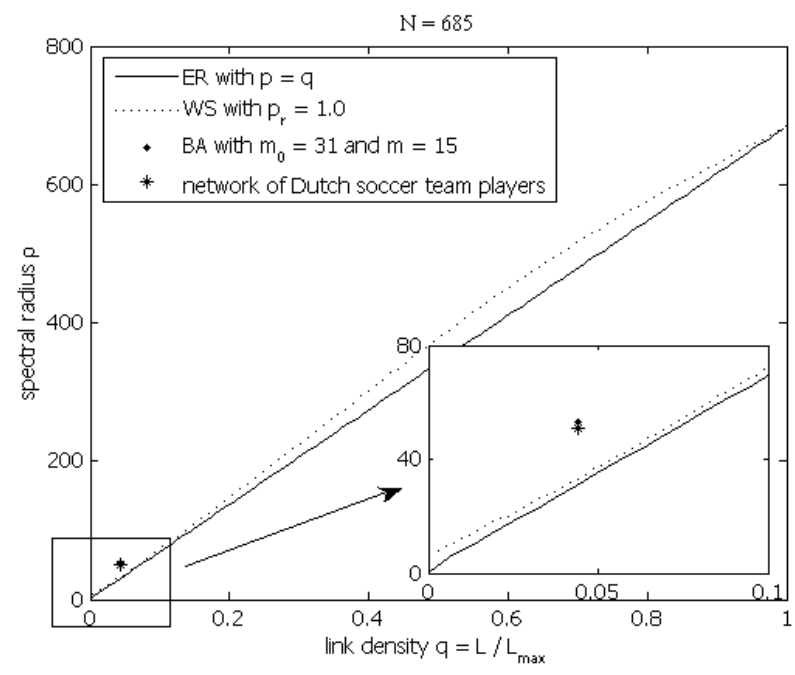

Fig. 2. Comparison between the spectral radius of the real-world network of Dutch soccer team players and the spectral radius of networks models: the random graph of Erdös-Rényi, the small-world graph of Watts-Strogatz and the scale-free graph of Barabási-Albert.

the topology, in most real-world networks, there is a relatively short path between any two nodes. The diameter $D$, presented in Table I, aims to illustrate this effect. Initially, the WS graph is built on the ring lattice $C(N, k)$, where each of the $N$ nodes is connected to its first $2 k$ neighbors ( $k$ on either side). Subsequently, a small-world is created by moving, for every node, one end of each link (connected to a clockwise neighbor) to a new location chosen uniformly with rewiring probability $p_{r}$, except that no double links or loops are allowed. The number of links $L$ in the WS graph, irrespective of $p_{r}$, is always equal to $L=N k$. Hence, the link density is $q=\frac{2 k}{N-1}$.

The BA graph gives rise to a class of graphs with a power-law degree distribution. The BA graph is based on two ingredients, growth and preferential attachment of nodes, which implies that nodes with larger degree are more likely candidates for attachment of new nodes. The BA algorithm starts with a small number $m_{0}$ of fully-meshed nodes, followed at every time step by a new node attached to $m \leq m_{0}$ nodes already present in the system. After $t$ time steps this procedure results in a graph with $N=t+m_{0}$ nodes and
$L=\frac{m_{0}\left(m_{0}-1\right)}{2}+m t$ links. Hence, the link density is $q=$ $\frac{m_{0}\left(m_{0}-1\right)+2 m t}{n(n-1)}$.

Figure 2 compares $\rho(\mathbf{A})$ and the average value of $\rho$ for generic network models. Furthermore, in Table III we consider $\rho$, calculated for a graph of identical link density as the one in real-world networks under consideration.

Figure 2 illustrates that the value of $\rho_{B A}$ is closest to $\rho(\mathbf{A})$. Moreover, as shown in Table III, the same tendency is observed for network $\mathbf{C}$, whereas for network $\mathbf{D}$ the value of $\rho(\mathbf{D})$ is not consistent with any of the examined models. Finally, the Dutch road infrastructure (network B) is most likely a subgraph of a two-dimensional ${ }^{2}$ lattice graph, as found in [11].

\begin{tabular}{|l|l|l|l|l|}
\hline spectral radius & A & B & C & D \\
\hline$\rho$ & 50.7 & 3.5 & 14.2 & 110.8 \\
\hline$\rho_{E R}$ & 30.9 & 4.1 & 4.4 & 7.7 \\
\hline$\rho_{W S}$ & 32.9 & 5.8 & 5.2 & 10.3 \\
\hline$\rho_{B A}$ & 52.9 & 14.2 & 14.3 & 15.1 \\
\hline
\end{tabular}

TABLE III

THE SPECTRAL RADIUS OF NETWORK MODELS: THE RANDOM GRAPH OF

ERDÖS-RÉNYI, THE SMALL-WORLD OF WATTS-STROGATZ AND THE

SCALE-FREE GRAPH OF BARABÁSI-ALBERT. FOR THE SIMULATIONS OF

THE SPECTRAL RADIUS THE SAME NUMBER OF NODES AND LINKS IS USED AS IN THE REAL-WORLD NETWORKS UNDER CONSIDERATION.

From the simulation results, we saw for small and medium rewiring probabilities $p_{r}$ that the spectral radius $\rho_{W S}$ virtually corresponds to $\rho_{E R}$. For example, the difference in $\rho$ between the WS graph with $p_{r}=0.5$ and the ER graph, in the complete $q$-range, is hardly noticeable (not shown). However, for high rewiring probabilities $p_{r}$ (close to 1 ), $\rho_{W S}$ has the tendency not to converge to $\rho_{E R}$, see Figure 2 . Thus, the spectral properties of the WS graph with $p_{r}=1$ are not identical to those of the ER graph. In order to examine this unexpected behavior in more detail, we have conducted some additional simulations on graphs with a small number of nodes, i.e. $N=21$ nodes. To obtain the WS graph with the link density $q=0.5$ and $p_{r}=1$, we move $k=5$ links of a given node to a new location chosen randomly in the ring lattice. The resulting spectral radius, after each rewiring step, is depicted in Figure 3. In addition, Figure 3 contains the spectral radius of the ER graph with the same link density. The depicted values are obtained by averaging over 100 simulation runs. It is obvious that $\rho_{W S}$ of the WS graph (obtained after 21 rewiring steps) is larger than $\rho_{E R}$ of the ER graph. A possible explanation lies in the fact that for $p_{r}=1.0$, the WS graph is an approximation of the random graph with the constraint that each node has a minimum of $d_{\min }=k$ links. This implies that the degree distribution of the WS graph is skewed. Figure 4 shows clearly

\footnotetext{
${ }^{2}$ The spectral radius of the 2D-lattice with sizes $z_{1}$ and $z_{2}$ such that $N=$ $\left(z_{1}+1\right)\left(z_{2}+1\right)$ and $L=2 z_{1} z_{2}+\left(z_{1}+z_{2}\right)$ is

$$
\rho_{2 \text { D-Lattice }}=2 \cos \left(\frac{\pi}{z_{1}+2}\right)+2 \cos \left(\frac{\pi}{z_{2}+2}\right)<4
$$
}


that the degree distribution of the ER graph is not identical to the one of the WS graph, where indeed $d_{\min }=k$.

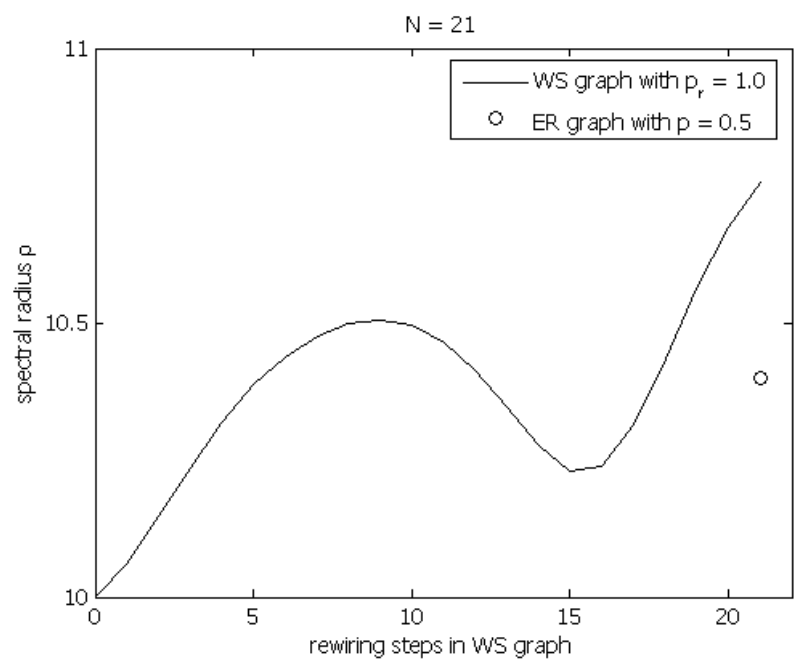

Fig. 3. The spectral radius of the small-world graph of Watts-Strogatz after, for every node, each link connected to a clockwise neighbor (i.e. here $k=5$ links) is rewired to a randomly chosen node with the probability $p_{r}=1.0$. At the end of $N$ steps, which correspond to the $N$ nodes, the spectral radius is compared with the one of the random graph of Erdös-Rényi, both for the link density $q=0.5$.

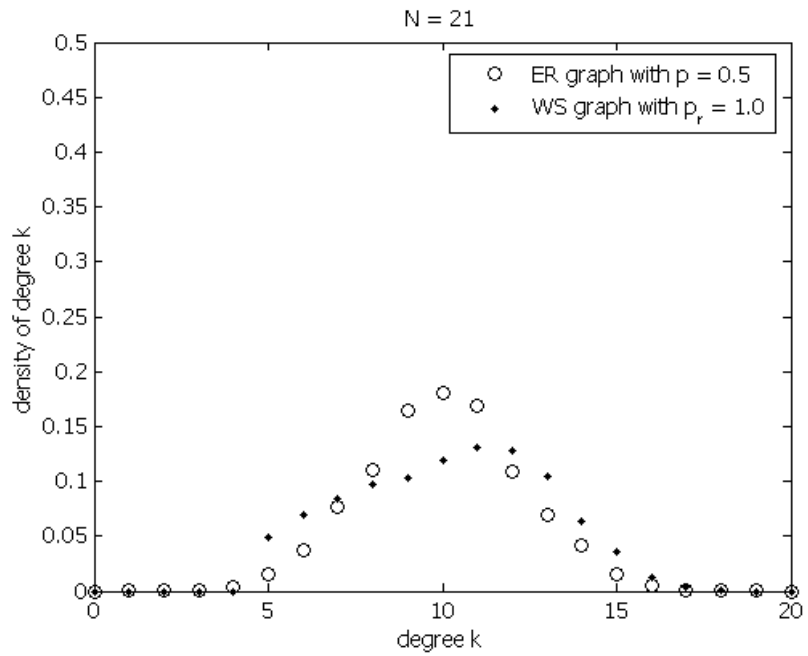

Fig. 4. The degree distribution of the small-world graph of Watts-Strogatz and the random graph of Erdös-Rényi, both for the link density $q=0.5$.

\section{CONCLUSION}

In this paper we have studied the spectral radius of a number of real-world networks. Our main conclusions are:

- for the Dutch soccer team network, the Dutch road map network and the Internet graph at router level, the upper bound given in [8] is reasonably tight
- for the Internet graph on AS level, all considered upper bounds seriously overestimate the spectral radius

- the spectral radii of the Dutch soccer team network and the Internet graph at the router level match well with those obtained from scale-free Barabási-Albert graphs with the same link density

- the spectral radius of the Dutch road map network matches well with that of a two dimensional lattice

- all considered network models, i.e. the random graph, small-world and scale-free graph give spectral radii that are much smaller than that of the Internet graph on AS level.

\section{ACKNOWLEDGEMENTS}

This research was supported by the Netherlands Organization for Scientific Research (NWO) under project number 643.000 .503 and by Next Generation Infrastructures (www.nginfra.nl). The authors thank Javier Martin Hernandez for writing a routine to generate BA networks.

\section{REFERENCES}

[1] R. Albert and A.-L. Barabasi, "Statistical mechanics of complex networks", Reviews of Modern Physics, 74 (1), 2002.

[2] B. Bollobás, "Random graphs", Cambridge University Press, 2001.

[3] A.-L. Barabasi, "Linked, The new science of networks", Perseus, Cambridge, MA, 2002.

[4] D. Cao, Bounds on eigenvalues and chromatic numbers, Linear Alg. Appl. 270, 1998.

[5] S.M. Cioabă, D.A. Gregory, and V. Nikiforov, Extreme eigenvalues for nonregular graphs, J. Combin. Th. B (2006), doi:10.1016/j.jctb.2006.07.006

[6] D.M. Cvetkovic, M. Doob, and H. Sachs, "Spectra of graphs, Theory and Applications", Johan Ambrosius Barth Verlag, Heidelberg, third edition, 1995.

[7] D.J. Daley and J. Gani, "Epidemic modelling: An Introduction", Cambridge University Press, 1999.

[8] K.C. Das, P. Kumar, Some new bounds on the spectral radius of graphs, Disc. Math. 281, 2004.

[9] Y. Hong, A bound on the spectral radius of graphs, Linear Alg. Appl. 108, 1988.

[10] Y. Hong, J.-L. Shu, K. Fang, A sharp upper bound on the spectral radius of graphs, J. Combin. Th. B 81, 2001.

[11] A. Jamakovic, H. Wang and P. Van Mieghem, "Topological Characteristics of the Dutch Road Infrastructure", Seminar Infrastructure Reliability TU Delft, June 22, 2006.

[12] A. Jamakovic and P. Van Mieghem, "The Laplacian Spectrum of Complex Networks", European Conference on Complex Systems, 2006.

[13] W. Mühlbauer, A. Feldmann, O. Maennel, M. Roughan, S. Uhlig, "Building an AS-Topology Model that Captures Route Diversity", Special Interest Group on Data Communication (SIGCOMM), 2006.

[14] M.E.J. Newman, "The structure and function of complex networks", SIAM Review 45 (2), 2002.

[15] P. Van Mieghem, "Performance Analysis of Communication Systems and Networks", Cambridge University Press, 2006.

[16] Y. Wang, D. Chakrabarti, C. Wang and C. Faloutsos, "Epidemic spreading in real networks: An eigenvalue viewpoint", 22nd Symposium in Reliable Distributed Computing, Florence Italy, Oct. 6-8, 2003.

[17] D.J. Watts, "Small-Worlds: The dynamics of networks between order and randomness", Princeton University Press, 1999. 